\title{
Survival of Mycobacterium abscessus isolated from people with cystic fibrosis in artificially generated aerosols
}

\author{
To the Editor:
}

Non-tuberculous mycobacterium (NTM) are increasingly found in the sputum of people with cystic fibrosis (CF), both in Europe and North America [1]. Specifically, Mycobacterium abscessus has emerged as a potentially important pathogen, with evidence of accelerated lung function decline [2]. Studies from two CF centres have found evidence of cross-infection between individuals with CF [3, 4], whereas studies from other centres have not replicated this finding [5-7]. M. abscessus has been isolated from household water and has been previously isolated from shower aerosols of people with pulmonary NTM disease [8, 9].

The aim of this study was to determine whether NTM could survive within artificially generated aerosols using a previously described laminar airflow model [10].

Four clinical isolates of M. abscessus, one clinical isolate of Mycobacterium chelonae and a reference stain of M. abscessus (NCTC 13031/ATCC 19977) were studied. The clinical NTM strains were isolated from individuals with CF attending a regional CF Centre. All NTM isolates were identified at a reference laboratory using a commercial kit (GenoType Mycobacterium; Hain Lifescience GmbH, Nehren, Germany). Isolates confirmed as M. abscessus were sub-speciated by PCR and sequencing of hsp65 and rpoB targets. Genotyping to identify strain clusters was performed using variable number tandem repeat (VNTR) based on the method of Harris et al. [11]. The M. abscessus massiliense strains studied were all isolated from unique individuals with CF. The strain of M. abscessus abscessus (VNTR type ST26) studied had been isolated from more than one individual in our CF cohort. All clinical isolates were associated with chronic infection as defined by American Thoracic Society/Infectious Diseases Society of America criteria [12].

All strains were examined in a laminar airflow model as previously described within a negatively pressurised Class II aerobiological chamber [10]. Aerosols were generated using a Collison 3-jet nebuliser (BGI, Butler, NJ, USA) containing suspensions of bacteria within $100 \mathrm{~mL}$ one-quarter strength Ringer's solution. The concentration of bacteria within the nebuliser suspension was determined both pre- and post-nebulisation using serial dilution.

The aerosols were delivered into a 110-mm diameter air-tight pipe with a variable length. In order to prevent cross-contamination, the pipe was sterilised by cleaning thoroughly with Virkon solution before each experiment. The pipe was then vented with sterile air via a high-efficiency particulate arrestance (HEPA) filter for $30 \mathrm{~min}$ before each experiment. Steady state conditions were ensured by allowing the apparatus to run for $10 \mathrm{~min}$ prior to air sampling. During each sampling event, $56.6 \mathrm{~L}$ of air was drawn through an Andersen 6-stage impactor (Andersen Inc, Idaho Falls, ID, USA) containing nutrient agar plates. The plates were then incubated and the concentration of viable bacteria in the air sample was determined. During experimentation, the length of the laminar flow apparatus was varied, and five air samples were taken at lengths of $2 \mathrm{~m}$ and $4 \mathrm{~m}$, which equates to aerosol ages of $40.3 \mathrm{~s}$ and $80.6 \mathrm{~s}$ respectively. In order to determine the size distribution of the droplet nuclei generated, Stages 1-6 of the Andersen sampler were used at all lengths of the model.

Statistical analysis was undertaken using GraphPad (Version 6.07; GraphPad Inc, La Jolla, CA, USA).

All strains of NTM were able to survive in particles of $\leqslant 2 \mu \mathrm{m}$ in diameter within the artificially generated aerosols (figure 1a). All of the strains studied were able to survive for $80.6 \mathrm{~s}$ and travel $4 \mathrm{~m}$ within the aerosols (figure 1b). There was a semi-log relationship between the concentration of NTM in the nebuliser suspension and the concentration of viable organisms in the aerosol $\left(\mathrm{R}^{2}=0.8728\right)$ (figure $1 \mathrm{~b}$ ).

This study demonstrates that NTMs can survive within aerosolised droplet nuclei particles within the respirable size range. The particle size distribution of the aerosols within this model was smaller than that demonstrated to be produced during coughing by individuals with CF by WAINwRIGHT et al. [13]. It would be important to demonstrate that people with CF can produce aerosols containing these pathogens. The behaviour of NTMs within respiratory secretions from people with CF may be different to that demonstrated in these artificially generated aerosols. The ability of NTMs to survive within this model appeared superior to that of $P$. aeruginosa. Different $P$. aeruginosa strains at a concentration of $10^{6} \mathrm{CFU} \cdot \mathrm{mL}^{-1}$ in one-quarter strength Ringer's solution produced aerosols containing less than $40000 \mathrm{CFU} \cdot \mathrm{m}^{-3}$ of viable bacteria [10]. We subsequently demonstrated these strains of $P$. aeruginosa could survive for at least 40 min within droplet nuclei in a different aerobiological model [14]. 


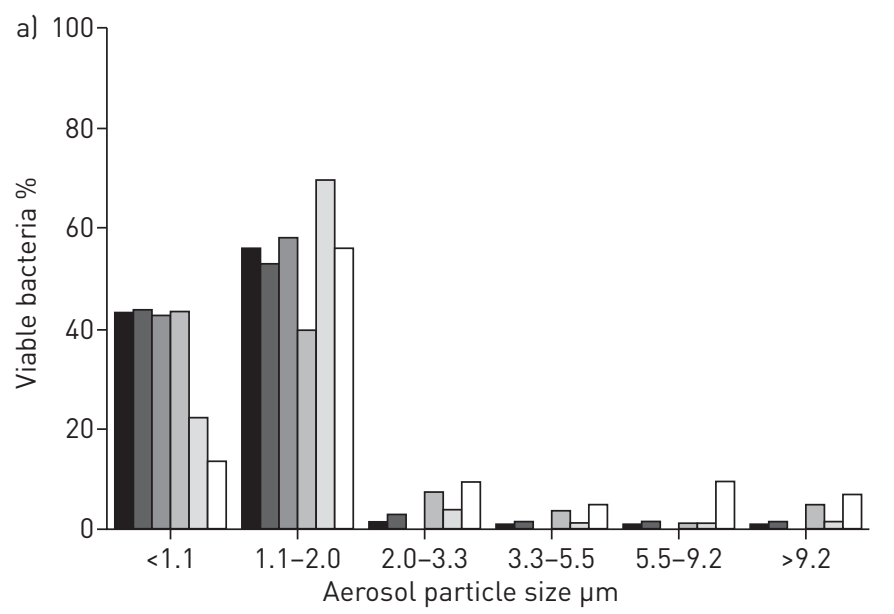

M. abscessus (NCTC 13031 /

ATCC 19977)

M. abscessus abscessus

(Type ST26)

M. chelonae (7621331)

M. abscessus massiliense

[7620169]

M. abscessus massiliense

(7621328)

M. abscessus massiliense

(7621752)

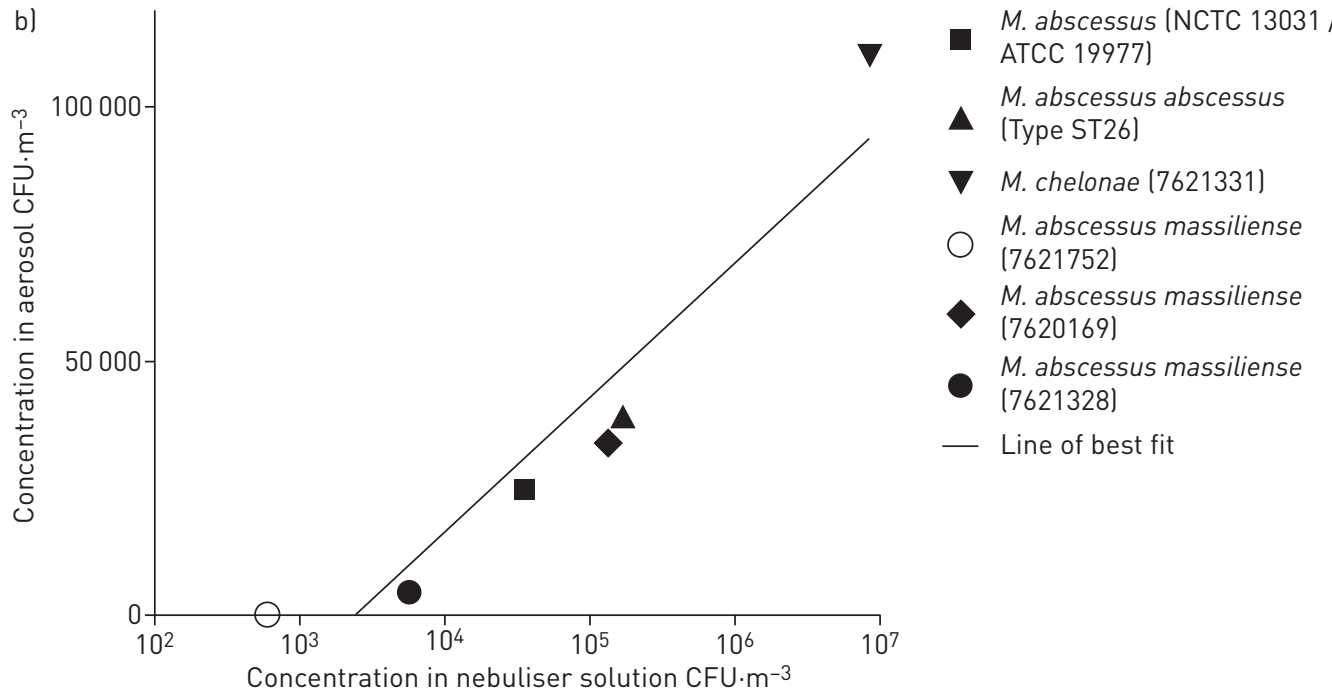

FIGURE 1 al Size distribution of aerosol particles containing non-tuberculous mycobacterium in the laminar air flow model. b) Relationship between concentration of viable bacteria within an aerosol age of $81 \mathrm{~s}$ and concentration in nebuliser solution. M. abscessus: Mycobacterium abscessus; M. chelonae: Mycobacterium chelonae.

All strains appeared to have similar characteristics in terms of airborne survival. There does seem to be a relationship between organism load in the nebuliser and concentration in the aerosol. This raises the possibility that individuals with high mycobacterial load in the sputum may represent a higher risk of generating potentially infectious aerosols. WAINWRight et al. [13] demonstrated that the higher concentrations of bacteria within sputum was associated with a greater concentration of bacteria within aerosols proceeded from people with CF during coughing.

BRYANT et al. [4] did not demonstrate a common environmental source of their outbreak despite extensive sampling, but air samples in clinical areas were not taken. They postulated that an airborne route of cross-infection may be possible and these data would support this hypothesis. This has important implications for the care of people with $\mathrm{CF}$ and reinforces the need for strict infection control practices. In response to the outbreak, the group at the Papworth CF centre (Cambridge, UK) have introduced segregation of individuals infected with $M$. abscessus in the out-patients environment and the use of negative pressure rooms for in-patient stays.

This study is limited due to the small number of strains studied, and further work needs to be undertaken to examine the survival of other mycobacterial and bacterial pathogens important to the care of people with CF within the air in both laboratory and clinical conditions. This may then lead to the development of strategies and interventions that may reduce the risk of cross-infection of harmful pathogens between people with CF.

@ERSpublications

M. abscessus survives aerosolisation suggesting airborne transmission between people with $\mathrm{CF}$ may occur http://ow.ly/K2x6302JpAW 
Louise A. Fletcher ${ }^{1}$, Yang Chen ${ }^{1}$, Paul Whitaker ${ }^{2}$, Miles Denton ${ }^{3}$, Daniel G. Peckham ${ }^{2}$ and Ian J. Clifton ${ }^{2}$ ${ }^{1}$ Dept of Civil Engineering, University of Leeds, Leeds, UK. ${ }^{2}$ Regional Adult Cystic Fibrosis Unit, St James's University Hospital, Leeds, UK. ${ }^{3}$ Dept of Microbiology, Leeds General Infirmary, Leeds, UK.

Correspondence: Ian J. Clifton, Regional Adult Cystic Fibrosis Unit, St James's University Hospital, Beckett Street, Leeds, LS9 7TF, UK. E-mail: i.clifton@nhs.net

Received: April 292016 | Accepted after revision: July 222016 | First published online: Sept 222016

Conflict of interest: None declared.

\section{References}

1 Floto RA, Olivier KN, Saiman L, et al. US Cystic Fibrosis Foundation and European Cystic Fibrosis Society consensus recommendations for the management of non-tuberculous mycobacteria in individuals with cystic fibrosis. Thorax 2016; 71: Suppl 1, i1-i22.

2 Esther CR Jr, Esserman DA, Gilligan P, et al. Chronic Mycobacterium abscessus infection and lung function decline in cystic fibrosis. J Cyst Fibros 2010; 9: 117-123.

3 Aitken ML, Limaye A, Pottinger P, et al. Respiratory outbreak of Mycobacterium abscessus subspecies massiliense in a lung transplant and cystic fibrosis center. Am J Respir Crit Care Med 2012; 185: 231-232.

4 Bryant JM, Grogono DM, Greaves D, et al. Whole-genome sequencing to identify transmission of Mycobacterium abscessus between patients with cystic fibrosis: a retrospective cohort study. Lancet 2013; 381: 1551-1560.

5 Bange FC, Brown BA, Smaczny C, et al. Lack of transmission of Mycobacterium abscessus among patients with cystic fibrosis attending a single clinic. Clin Infect Dis 2001; 32: 1648-1650.

6 O’Driscoll C, Konjek J, Heym B, et al. Molecular epidemiology of Mycobacterium abscessus complex isolates in Ireland. J Cyst Fibros 2016; 15: 179-185.

7 Harris KA, Underwood A, Kenna DT, et al. Whole-genome sequencing and epidemiological analysis do not provide evidence for cross-transmission of Mycobacterium abscessus in a cohort of pediatric cystic fibrosis patients. Clinl Infect Dis 2015; 60: 1007-1016.

8 Thomson R, Tolson C, Carter R, et al. Isolation of nontuberculous mycobacteria (NTM) from household water and shower aerosols in patients with pulmonary disease caused by NTM. J Clin Microbiol 2013; 51: 3006-3011.

9 Thomson R, Tolson C, Sidjabat H, et al. Mycobacterium abscessus isolated from municipal water: a potential source of human infection. BMC Infect Dis 2013; 13: 241.

10 Clifton IJ, Fletcher LA, Beggs CB, et al. A laminar flow model of aerosol survival of epidemic and non-epidemic strains of Pseudomonas aeruginosa isolated from people with cystic fibrosis. BMC Microbiol 2008; 8: 105.

11 Harris KA, Kenna DT, Blauwendraat C, et al. Molecular fingerprinting of Mycobacterium abscessus strains in a cohort of pediatric cystic fibrosis patients. J Clin Microbiol 2012; 50: 1758-1761.

12 Griffith DE, Aksamit T, Brown-Elliott BA, et al. An official ATS/IDSA statement: diagnosis, treatment, and prevention of nontuberculous mycobacterial diseases. Am J Respir Crit Care Med 2007; 175: 367-416.

13 Wainwright CE, France MW, O'Rourke P, et al. Cough-generated aerosols of Pseudomonas aeruginosa and other Gram-negative bacteria from patients with cystic fibrosis. Thorax 2009; 64: 926-931.

14 Clifton IJ, Fletcher LA, Beggs CB, et al. An aerobiological model of aerosol survival of different strains of Pseudomonas aeruginosa isolated from people with cystic fibrosis. J Cyst Fibros 2010; 9: 64-68.

\section{The impact of exposure to particulate air pollution from non-anthropogenic sources on hospital admissions due to pneumonia}

To the Editor:

Community-acquired pneumonia is a significant cause of morbidity and mortality among older adults [1]. The role of air pollution as a risk factor for pneumonia hospitalisations and mortality has been investigated [2,3] with most evidence coming from studies in North American and European cities, where anthropogenic sources are predominant in generating air pollution.

Particulate non-anthropogenic air pollution originating from dust is a common public health risk. Being located between the Sahara and the Arabian deserts (the world's largest dust-belt), the Negev region of Israel is exposed to extremely high levels of particulate matter originating from natural dust storms. During dust storms in this region, particulate matter levels can significantly exceed those defined as acceptable in terms of air quality and human health $\left(50 \mu \mathrm{g} \cdot \mathrm{m}^{-3}\right)$ with hourly concentrations of $100-5000 \mu \mathrm{g} \cdot \mathrm{m}^{-3}[4]$. Dust particles reach the southeastern Mediterranean by two main trajectories: one from the west (North 